\section{Beyond the parish}

\section{T.C. Champion}

Journal of World Prehistory. Co-editors Fred Wendorf and Angela E. Close. Plenum. 4/yr. US \$95, elsewhere $\$ 110$.

THE GROWTH in archaeological research throughout the world shows no sign of slowing down. One of the consequences of this information explosion has been something of a bibliographical nightmare.

Most research is still designed and carried out in a local context, and the results usually appear in regional or national journals, or monograph series. The multiplicity of languages used means that even the most gifted linguists among us cannot follow the literature, even assuming that we know what we are looking for and that the library can provide it. There are few truly international journals, and few regions where an effective abstracting service exists. This is particularly ironic at a time when archaeologists are increasingly interested in general patterns of social organization and general processes of change, rather than merely local sequences of culture history. The archaeologist working on general topics is therefore at a disadvantage, and it is all too easy to overlook relevant work through simply never knowing of its existence.

So there is a desperate requirement for better information services in archaeology, one of them being the need for a supply of reliable review articles. Until recently this service was to a large extent

\section{Test-tube toxins}

\section{T.A. Connors}

Toxicology in Vitro. Editors I.F.H. Purchase and G.C. Hard. Pergamon. 4/yr. $£ 70, \$ 110$ (institutional); individual rates also available.

Comments on Toxicology. Editors A. Wallace Hayes and William O. Berndt. Gordon \& Breach. 6 issues per volume (see below). $\$ 268$ (corporate); $\$ 190$ (library); $\$ 80$ (personal).

Toxicology covers a broad area, involving not only research scientists of different disciplines, but industrialists concerned with safety evaluation and government bodies responsible for the regulation of the use of chemicals. Subsections of the subject are becoming more clearly defined, and have been marked by the appearance over the past few years of journals such as Human Toxicology, Reproductive Toxicology and the recently announced Chemical Research in Toxicology, a publication of the American provided by Academic Press's annual series Advances in World Archaeology, which, I understand, was discontinued after the appearance of Vol. 5. Now we have a new journal under the same editorship, but from a different publisher. Journal of World Prehistory very much retains the same style and contents as the book series, which makes it rather unusual for a journal. It includes nothing but review articles, two per issue in the first volume, thus giving authors 50 pages or more for detailed and critical discussion of recent research, and enabling them to include good bibliographies.

The journal covers prehistoric archaeology only, and within that the main interest seems to lie in the earlier periods and less complex societies. Geographical coverage is extensive. The first volume included papers on Europe, South America, northern Asia and island southeast Asia, as well as one on the techniques and potential of radiocarbon accelerator dating; they are generally well illustrated, though occasionally reproduction from original sources has given indifferent results, and the retention of untranslated captions in some figures looks rather odd.

There is no doubt that, for the good of the subject, such an outlet for reviews is needed, and the quality of the first volume of Journal of World Prehistory augurs well for its future. It would be a sad day if archaeologists were too obsessed with their own parochial interests to take advantage of a venture such as this.

T.C. Champion is Senior Lecturer in the Department of Archaeology, University of Southampton, Southampton SO9 $5 \mathrm{NH}, U K$

toxicity testing in whole animals.

No new journal can be expected to publish high-quality work only, and the variation in scientific standard in the first two volumes is much what one would expect. The papers range from considerations of simple in vitro models for quantifying the properties of, for example, skin irritants, to more basic studies on mechanisms of action. As long as this balance is maintained then the journal should flourish. The stress will be on the publication of research papers, but brief communications will also be considered, and it is pro-

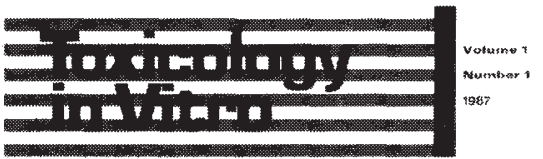

posed to include concise interpretative reviews from time to time. Publication speed is reasonable and the quality of printing, reproduction of figures and so on is similar to that of other Pergamon scientific journals - that is, adequate but not outstanding.

It is a much more difficult task to predict the future of Comments on Toxicology, because the journal is not really well known at present, amongst British toxicologists at least. Its aims are to present topical issues in a manner designed to stimulate discussion and critical examination. Because of the complexity of toxicology, there is certainly a need for a forum of this sort, and the journal has indeed published some interesting material - for instance the five papers in Vol.II, No.2, devoted to a discussion of the importance of blind-slide reading in carcinogenicity and chronic toxicity studies. The general idea is that each chosen subject should be introduced by a guest editor who will set the stage for the theme under consideration. One criticism, however, is that this plan is not working too well at the moment; some numbers have not had guest editors, while the introductions to others have been short and unincisive.

I hope Comments on Toxicology succeeds because the idea behind it is a good one. But those libraries with diminishing budgets might feel it too much of a luxury and restrict their purchases to journals containing reports of original research. $\square$

T.A. Connors is Director of the MRC Toxicology Unit, Medical Research Council Laboratories, Woodmansterne Road, Carshalton, Surrey SMS $4 E F, U K$.

The journal does not over-emphasize the development of alternatives to the use of animals, but a statement in the editorial of the first number makes it clear that one of its principal aims is to stimulate an improvement in the hazard assessment of chemicals using in vitro procedures. There is, however, the recognition that in vitro methods for the most part remain complementary to in vivo systems and may often be used for pre-screening prior to
- Harwood Academic Publishers and Gordon and Breach Science Publishers produce journals under the 'flow system'. Once sufficient papers are accepted to complete an issue, that issue is closed and quickly published. According to the publishers, this avoids the usual delays created by rigid schedules, but means that the number of issues per year cannot be accurately predicted. 